\title{
Korrekter Umgang mit Geschenken und Einladungen zum Mittagessen
}

\author{
Bruno Henggi ${ }^{a}$, Charlotte Schweizer ${ }^{b}$

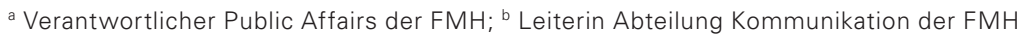

Am 1.1.2020 treten neue Vorschriften betreffend den Umgang mit Heilmitteln in Kraft. Die neuen Bestimmungen bringen strengere Regelungen mit sich, welche direkt für den Berufsalltag von Ärztinnen und Ärzten relevant sind. Sie sind in der Verordnung «Integrität und Transparenz im Heilmittelbereich» (VITH) festgehalten. Diese hat zum Ziel, die Integrität im Umgang mit Arzneimitteln zu stärken und dessen Transparenz zu erhöhen. Daher reguliert sie Preisrabatte und Abgeltungen seitens Arzneimittellieferanten stark und detailliert. Weiter enthält sie Bestimmungen über Unterstützungsbeiträge im Bereich Forschung sowie Weiterund Fortbildung. Auch für die Annahme von Geschenken oder Einladungen zum Essen gibt es strikte Vorschriften.

Für Ärztinnen und Ärzte bedeutet dies, dass sie sich mit den Änderungen der neuen Verordnung vertraut machen müssen, um die neuen Bestimmungen achtsam und sorgfältig umsetzen zu können. Weiter müssen Ärztinnen und Ärzte ihre diesbezüglichen Handlungen konsequent und umfassend dokumentieren. Als Berufsverband erachtet es die FMH als ihre Pflicht, ihre Mitglieder auf die strengere Regelung aufmerksam zu machen und sie kontinuierlich und regelmässig über die neuen gesetzlichen Bestimmungen zu informieren. Dies möchten wir in anschaulicher Form in einer Artikelserie in vier Teilen realisieren.

Im vorliegenden Beitrag gehen wir näher auf Fragen ein, welche sich im Zusammenhang mit Geschenken und Einladungen zum Mittagessen stellen. Wir behandeln diese anhand von praktischen Beispielen, wie sie sich im Berufsalltag von Ärztinnen und Ärzten ergeben können.

\section{Einladungen zum Mittagessen}

Frage: Eine Fachperson erhält von einem Pharmaunternehmen eine Einladung zum Mittagessen. Nun stellt sich die Frage, unter welchen Voraussetzungen eine solche Einladung angenommen werden darf bzw. unter welchen Bedingungen die Übernahme der Verpflegungskosten durch das Unternehmen gesetzeskonform ist.
Antwort: Beim erwähnten Mittagessen muss es sich um ein Fachgespräch handeln. Dies bedeutet, dass ein Thema Gegenstand des Gesprächs ist, welches für das Pharmaunternehmen von Bedeutung ist und somit einen Mehrwert generiert. Genauer ist das «Fachgespräch» juristisch noch nicht definiert. Klar ist, dass mit dem Fachgespräch eine fachliche Gegenleistung verbunden sein muss. Dafür dürfen Ärztinnen und Ärzte eine Einladung mit einem Gegenwert von bis zu 100 CHF annehmen. Einladungen, die einen Wert von 100 CHF überschreiten, sind nicht zulässig, ausser sie werden dokumentiert mittels einer schriftlichen Vereinbarung, welche die Leistung und die Gegenleistung festhält. Damit die Schwelle von 100 CHF eingehalten wird, empfiehlt es sich, die Preise auf der Speisekarte und allenfalls der Weinkarte zu beachten. Zur Sicherheit können Fachpersonen zur eigenen Dokumentation auch Gesprächsnotizen des Mittagessens erstellen.

\section{Gesetzliche Grundlage VITH: Erläuterung}

Ärztinnen und Ärzte dürfen also Einladungen zum Mittagessen ohne schriftliche Vereinbarung annehmen und sich ihre Mahlzeit bezahlen lassen, sofern es im Rahmen eines Fachgesprächs stattfindet und die Kosten inklusive Getränken höchstens 100 CHF betragen. Einladungen zum Mittagessen sind unzulässig, 
wenn sie ohne fachliches Gespräch als Gegenleistung stattfinden oder wenn der Wert der Mahlzeit inklusive Getränken den Wert von 100 CHF übersteigt und keine schriftliche Vereinbarung abgeschlossen wurde, welche die Leistung und Gegenleistung dokumentiert.

\section{Verordnungstext}

\section{Art. 7 Abgeltungen für gleichwertige Gegenleistungen}

1 Als Abgeltungen für gleichwertige Gegenleistungen einer Fachperson oder Organisation nach Artikel 55 Absatz 2 Buchstabe c HMG zulässig sind Abgeltungen, die:

a. auf einer schriftlichen Vereinbarung basieren, woraus Art und Umfang von Gegenleistung und Abgeltung hervorgeht; und

b. in einem angemessenen Verhältnis zur Gegenleistung stehen.

2 Im Rahmen eines Fachgesprächs ist die Übernahme von Verpflegungskosten bis höchstens 100 Franken von der Verpflichtung nach Absatz 1 Buchstabe a ausgenommen.

\section{Geschenke und Vergütungen}

Frage: Unter welchen Bedingungen darf eine Fachperson ein Geschenk von einem Pharmaunternehmen annehmen?

Antwort: Geschenke ohne Bezug zur medizinischen oder pharmazeutischen Praxis sind explizit verboten. Darunter fallen zum Beispiel Geschenkgegenstände wie Wein oder Spirituosen, aber auch Einladungen oder Eintrittskarten zu kulturellen Anlässen. Zulässig sind jedoch Geschenke von bescheidenem Wert, die für die medizinische oder pharmazeutische Praxis von Belang sind. Dies bedeutet einerseits, dass der Wert des Geschenks pro Fachperson oder Arztpraxis (bei mehreren Fachpersonen) 300 CHF pro Jahr nicht überschreiten darf. Bei Einzelpraxen sind dies 300 CHF pro Arzt und Jahr. Bei Gruppenpraxen gelten die $300 \mathrm{CHF}$ als gemeinsame kumulierte Obergrenze für alle Ärztinnen und Ärzte derselben Praxis. Andererseits muss das Geschenk im Zusammenhang mit der Berufsausübung der Fachperson stehen oder Patientinnen und Patienten direkt zugutekommen. Zulässig sind zum Beispiel:

- Fachliteratur oder Weiter- und Fortbildung mittels Einsatz von elektronischen Medien wie Bild-, Tonund Datenträgern

- Arbeitsgeräte wie Fiebermesser, Computer-Software oder Mobiltelefone für den Notfalldienst (auch Mobiltelefone dürfen nicht mehr als 300 CHF kosten)
- Praxisausstattung wie Wasserspender mit Mineralwasser für die Patientinnen und Patienten oder Produkte fürs Wartezimmer wie beispielsweise Lektüre oder Spielwaren für Kinder. Dabei ist jedoch zu beachten, dass Arzneimittellieferanten versuchen könnten, Geschenkgegenstände gleichzeitig als Werbeträger einzusetzen und Ärztinnen und Ärzten dafür eine angeblich legale Entschädigung auf vertraglicher Basis anzubieten. Wir warnen vor solchen Angeboten, da das Geschenk in diesem Fall den beschriebenen Ausnahmebedingungen nicht mehr entspricht. Wir empfehlen, solche Angebote juristisch prüfen zu lassen.

Diese erwähnten zulässigen Geschenke, die für die medizinische oder pharmazeutische Praxis von Belang sind, müssen nicht schriftlich dokumentiert werden.

\section{Konkrete Beispiele von Geschenken zur Veranschaulichung}

Kinderbilderbuch: Ein Arzt erhält für das Wartezimmer ein neues Kinderbilderbuch zum Thema Cholesterin.

Frage: Darf er dieses Geschenk annehmen?

Antwort: Ja, dieser Vorteil kommt den Patientinnen und Patienten zugute. Der Arzt muss das Buch jedoch im Wartezimmer auflegen.

Schaukelpferd: Für das Wartezimmer erhält eine Ärztin ein Schaukelpferd zum Spielen für die Kinder ihrer Patientinnen und Patienten. Auf dem Pferd steht «Made in Switzerland», und es hat eine Etikette, auf der steht «Dieses Produkt wurde von Hand gefertigt und entspricht höchster Schweizer Qualitätsarbeit».

Frage: Dürfen Ärztinnen und Ärzte dieses Geschenk annehmen?

Antwort: Ja, dieser Vorteil kommt den Patientinnen und Patienten zugute. Der Arzt muss das Schaukelpferd jedoch im Wartezimmer zur Benutzung zur Verfügung stellen.

Gutschein: Eine Ärztin gewinnt am Kongress eines Pharmaunternehmens einen Gutschein für eine Übernachtung im Hotel Mont Cervin in Zermatt.

Frage: Darf die Ärztin diesen Gutschein annehmen? Antwort: Nein, dieser im Rahmen eines Wettbewerbs gewonnene Vorteil ist unzulässig, da er nicht bescheiden und auch nicht für die medizinische oder pharmazeutische Praxis von Belang ist. Zulässig im Rahmen eines Wettbewerbs wäre beispielsweise die Annahme von Fachliteratur im Wert von unter 300 CHF pro Arztpraxis und Jahr. 


\section{Besuche von Pharmavertretern}

Ein Vertreter eines Pharmaunternehmens stellt im Rahmen eines Praxisbesuchs die neusten Medikamente vor. Er bringt für alle Praxisangestellten Backwaren mit.

Frage: Darf diese Aufmerksamkeit angenommen werden?

Antwort: Auch Personen, die nichteigenverantwortlich Arzneimittel abgeben, sowie Praxisangestellte (MPA) fallen unter die Vorteilsbestimmungen. Daher werden solche Mitbringsel als problematisch eingestuft. Wenn ein Vertreter Backwaren mitbringt, sollten diese im Wartezimmer den Patientinnen und Patienten angeboten oder ganz abgelehnt werden.

\section{Gesetzliche Grundlage VITH: Erläuterung}

Der Zweck der neuen gesetzlichen Grundlage ist, zu vermeiden, dass die Verschreibung, Abgabe, Anwendung oder der Einkauf von verschreibungspflichtigen Arzneimitteln durch Zuwendungen jedweder Art beeinflusst wird. Anstatt ein vollständiges Verbot von Zuwendungen durchzusetzen, definiert die neue Verordnung einen Ausnahmekatalog, der abschliessend auflistet, was rechtlich weiterhin möglich sein soll. Darunter fallen sogenannte Vorteile von bescheidenem Wert. Diese sind begrenzt auf 300 CHF pro Jahr und Fachperson respektive Arztpraxis. Bei Gruppenpraxen gelten die 300 CHF als Obergrenze für alle in der Praxis arbeitenden Ärztinnen und Ärzte zusammen. Und wichtig: Sie müssen für die medizinische Praxis von Belang sein. Das können sein: Fachliteratur, Instrumente wie Fiebermesser, Computer-Software oder ein Mobiltelefon für den Notfalldienst. Darunter fallen auch Wasserspender mit Mineralwasser für die Patienten, Spielzeug für Kinder oder Zeitschriften für das Wartezimmer. Persönliche Geschenke jeglicher Art sind unzulässig, wenn sie nicht im Zusammenhang mit der Berufsausübung stehen und somit nicht den Patientinnen und Patienten zugutekommen.

Gewinne und Preise im Rahmen von Wettbewerben sind nur dann zulässig, wenn sie einen bescheidenen Wert haben und von Belang für die medizinische und pharmazeutische Praxis sind. Ausserdem darf sich der Wettbewerb nur an den Adressatenkreis von Fach- werbung richten, und die Wettbewerbsteilnahme darf nicht an den Einkauf von verschreibungspflichtigen Arzneimitteln geknüpft sein.

\section{Verordnungstext}

\section{Abschnitt: Integrität}

\section{Art. 3 Vorteile von bescheidenem Wert}

1 Als Vorteile von bescheidenem Wert nach Artikel 55 Absatz 2 Buchstabe a HMG zulässig sind Vorteile an Fachpersonen im Gesamtwert von höchstens 300 Franken pro Fachperson und Jahr, die für die medizinische oder pharmazeutische Praxis von Belang sind.

2 Ein Vorteil ist für die medizinische oder pharmazeutische Praxis von Belang, wenn er in direktem Zusammenhang mit der Berufsausübung der Fachperson steht oder direkt der Kundschaft der Fachperson zugutekommt.

3 Gewinne und Preise im Rahmen von Wettbewerben sind nur zulässig, wenn:

a. sie aus einem Vorteil von bescheidenem Wert und von Belang für die medizinische oder pharmazeutische Praxis bestehen

b. der Wettbewerb sich ausschliesslich an den Adressatenkreis für Fachwerbung im Sinne von Artikel 3 der Arzneimittel-Werbeverordnung vom 17. Oktober 2001 (AWV) richtet; und

c. die Teilnahme am Wettbewerb nicht an den Einkauf von verschreibungspflichtigen Arzneimitteln geknüpft ist.

\section{Empfehlung}

Als Fazit empfiehlt die FMH Ärztinnen und Ärzten, bei Arzneimittellieferungen künftig gewisse Vorsichtsmassnahmen zu treffen. Bezüglich Geschenken und Mitbringseln sollten Ärztinnen und Ärzte darauf achten, dass Geschenke ohne Bezug zur medizinischen und pharmazeutischen Praxis explizit verboten und daher abzulehnen sind. Geschenke dürfen den Wert von $300 \mathrm{CHF}$ pro Jahr und pro Fachperson oder Praxis nicht überschreiten.

Was Einladungen zum Mittagessen anbelangt, ist zu beachten, dass dieses höchstens einen Gegenwert von $100 \mathrm{CHF}$ inklusive Getränken haben darf und dass die Kostenlimite im Eigeninter esse der Ärztinnen und Ärzte im Zweifelsfall zu kontrollieren ist. 